\title{
User Evaluation of the SYNFACE Talking Head Telephone
}

\author{
Eva Agelfors $^{1, \star}$, Jonas Beskow ${ }^{1}$, Inger Karlsson ${ }^{1}$, Jo Kewley ${ }^{2}$, \\ Giampiero Salvi ${ }^{1}$, and Neil Thomas ${ }^{2}$ \\ ${ }^{1}$ Kungl Tekniska Högskolan (KTH), Dept of Speech, Music and Hearing, \\ SE-100 44 Stockholm, Sweden \\ \{agelfors, beskow, inger, giampi\}@kth.se \\ ${ }^{2}$ Royal National Institute for Deaf People (RNID), London EC1Y 8SL, UK \\ neil.thomas@rnid.org.uk
}

\begin{abstract}
The talking-head telephone, Synface, is a lip-reading support for people with hearing-impairment. It has been tested by 49 users with varying degrees of hearing-impaired in UK and Sweden in lab and home environments. Synface was found to give support to the users, especially in perceiving numbers and addresses and an enjoyable way to communicate. A majority deemed Synface to be a useful product.
\end{abstract}

\section{Introduction}

Hard of hearing people often rely on lip-reading to follow conversations. This works well in face-to-face situations but over the telephone this visual information is missing, and people are left to rely only on what they can hear. This means that telephone conversations can be difficult and frequently these problems are greater when the person at the other end is a stranger. Many people with hearing difficulties report that they have stopped using the telephone as their hearing impairment has increased.

A solution a this problem could be video telephony. The current technology for video telephony lacks the quality that is necessary for lip reading [1, and sets a number of problems ranging from lack of privacy to the need for special equipment on both ends of the line. The SYNFACE project has developed a prototype system with a synthetic talking face that reproduces the lip movements of a talker only from the acoustic signal [2]. This can be used by the person with impaired hearing to improve understanding, transparent to the person on the other end of the line. The prototype has been developed for Swedish, English and Dutch. This paper describes user evaluations of the prototype system for Swedish and English with hearing impaired users in Sweden (KTH) and UK (RNID), respectively.

\section{SYNFACE Talking Head Telephone}

The main parts of the Synface system are a phoneme recogniser and a three dimensional artificial face that runs on a standard pc. As the automatic pho-

\footnotetext{
^ Authors in alphabetical order.
} 
netic recognition and the visual animation software need to run simultaneously on a compact machine, the computational resources are quite limited. A unique phoneme recogniser was developed for Synface [3] that was optimised to deliver phonetic output with very low latency $(30 \mathrm{msec})$. Recognisers for the three languages were trained on speech collected in the ESPRIT SpeechDat project. The three dimensional artificial face animation is based on work originally described in [4]. An articulatory control model [5] is used to animate the movements of the artificial face. This model takes time-stamped phonetic symbols as input and produces articulatory control parameter trajectories to drive the face model. The control model includes coarticulation to account for the influence of neighbouring segments. A special real-time version of the rule-based control model has been developed, that uses a finite time-window of articulatory anticipation. For the prototype Synface system, control models have been adapted to Dutch, English and Swedish.

In the Synface prototype used in the evaluations the incoming telephone speech is tapped of the line, fed into the computer and analysed to produce the face articulation control parameters. The speech is delayed by $200 \mathrm{msec}$ to allow for the delays in the recogniser and the artificial face, time aligned with the face movements and played back to the user in synchrony with the face.

\section{SYNFACE User Evaluations}

The focus of the evaluations was on gaining users attitudes and opinions towards Synface. The evaluations reported in the present paper were performed at two places: RNID performed the evaluations of the English prototype and KTH of the Swedish prototype. Between the two sites, a total of 49 evaluations have been completed. These can be broken down into lab-based and home based trials, as described below.

The structure of all tests were similar although the duration and locality differed. The evaluation participants were informed about how Synface worked, they performed some comprehension tests and made telephone calls using the Synface prototype. In connection to the tests the evaluation participants were asked to fill in a set of three questionnaires:

- Selection questionnaire. Designed to find out basic information about potential participants to ensure that a variety of users were recruited for the evaluations.

- Pre-Evaluation questionnaire. Designed to gain an understanding of each participant, how they use existing communication methods and the problems they have encountered.

- Post-Evaluation questionnaire. Designed to gain the participants' opinions of Synface, the problems they encountered, the usability of the prototype, whether they would use such a system in the future and additional marketing related questions. 
The questionnaires were compiled by RNID in English and translated into Swedish to facilitate comparisons between the evaluation results. In addition to filling in the questionnaires all participants were interviewed after the evaluation sessions.

\subsection{British Evaluations}

The evaluation participants were selected from a larger group who had volunteered to participate. They had filled in the selection questionnaire and were selected to form a group with an even spread of hearing impairments and a bias towards the older age groups.

Lab-Based Evaluations. Extensive lab based evaluations were carried out with 33 participants (14 males, 19 females). Of these, 25 used hearing aids, 5 had a cochlear implant (CI) and 3 participants used no listening devices. Participants were asked to describe their hearing level (when using hearing aids or CI, if applicable). Fig. 1 illustrates the distribution of participants' responses. To gauge if and how participants use lipreading they were asked a number of questions. $97 \%$ of participants stated that seeing a speaker's face helped them to understand conversations. $36 \%$ felt they were able to understand without seeing the talker's face if in a quiet room, whereas only $21 \%$ felt they would be able to in a noisy room. They also differed in how much they used voice telephones. Five participants no longer used a telephone, 4 participants used the telephone less than once a week and 4 participants used a voice telephone weekly. Only $48 \%$ of participants felt confident using a voice telephone. The main reason for not using the telephone was that they could not hear enough. Results also suggested that communication with familiar voices is easier than with strangers: $51.5 \%$ of participants felt that they could easily understand people they knew on the telephone whereas only $15.2 \%$ felt they could easily understand people they did not know. Several participants stated that they struggled understanding names, numbers and people with accents.

The evaluations were carried out in a research lab at the RNID, and the same experimenter carried out all the evaluations. In each lab evaluation participants

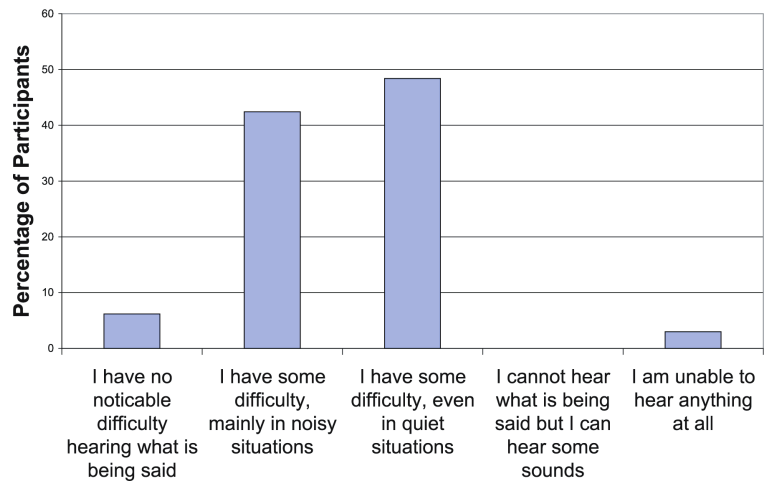

Fig. 1. British participants' descriptions of hearing loss with hearing aid or CI 
were first asked to complete the pre-evaluation questionnaire. They were then shown the Synface video and any questions in relation to Synface and the evaluation were answered. Participants were then shown the short story about the North Wind.

The experimenter then described each of the four scenarios that would be the topics of each of the calls they would make using the prototype. Each participant was given the same examples of possible questions they could ask. A second experimenter was at the other end of each of the calls. After participants had completed the scenarios they were asked to complete the Post-Evaluation questionnaire and then a full debriefing interview was carried out.

Home-Based Evaluations. The second part of the UK evaluations was to investigate how participants' attitudes and opinions changed with extended usage and whether users' ability to lip-read the face improved with practice. 6 people, selected from those that participated in the lab-based tests participated in these evaluations. These home-based evaluations involved the prototype being installed in a participant's home for a period of 2 weeks. The participants reported either mild or moderate hearing losses. Four of these participants stated that they find communication using a voice telephone "somewhat effective" and 2 stated that they find the telephone "not at all effective". Of these participants 3 use a voice telephone on a daily basis, 1 uses a voice telephone on a weekly basis, 1 uses a voice telephone less than once a week and 1 participant no longer uses a voice telephone. In addition to questionnaire results, data was collected using an informal interview at the start and end of each evaluation as well as a short telephone interview two weeks after the evaluation had ceased. Quantitative data was also obtained by giving participants a short questionnaire based on the post evaluation questionnaire used in the lab-based evaluations.

To gauge whether any learning effects took place, participants were scored on the number of keywords understood in pre-recorded sentences. These sentences were presented to participants at the start and end of each evaluation.

\subsection{Swedish Evaluations}

The Swedish evaluations were either lab-based, 5 participants, or home- or workbased, 5 participants. The lab-based evaluations lasted about half a day and contained different tasks including comprehension tests, a telephone call to one experimenter following a set scenario and unsupervised calls. The home- or workbased evaluations ran for one to four days. These evaluations contained some initial familiarisation with Synface and how it worked and a call to the same experimenter following the same scenario as in the lab-based evaluation, otherwise they were unsupervised. The home-based test participants also took part in a comprehension test at KTH after their evaluations.

The evaluation participants were recruited among patients at a cochlear implant clinic and a hearing rehabilitation clinic in Stockholm. All participants reported that they had problems understanding unknown people on the phone while one person from each group reported having problems understanding people they know. Their lipreading skills were estimated in the comprehension test, Fig 2. 


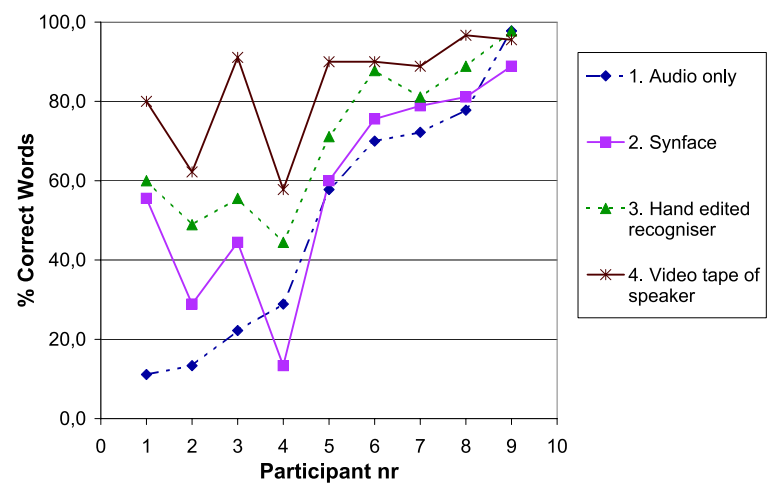

Fig. 2. Word comprehension of 1. speech only, 2. speech with Synface, 3. speech with hand edited talking head synthesis and 4. natural visual speech. All speech is from the same speaker. Results from the Swedish evaluations.

Lab-Based Evaluation. The five subjects, two women and 3 men, that participated in the KTH lab-based evaluations had some prior experience of the Synface technology. Four of the subjects used hearing aids equipped with t-coil for the telephone conversations. The fifth subject had a cochlear implant (CI). Two subjects stated that they could not hear what is being said but could hear some sounds, one that he had some difficulty, even in quiet situations and two had some difficulty, mainly in noisy situations when they were asked to describe their hearing level in the questionnaire. The telephone usage varied between the subjects, one person had stopped using the telephone two years ago, one person used a telephone weekly and the remaining three used it most days.

Home-Based Evaluation. Five evaluation participants, two males and three females, tested Synface in this series, one participant used Synface at work, the other four used it at home. Three of the participants used a CI, the other two used hearing aids. All had a severe to profound hearing loss. One participant stated that she had no difficult in hearing what is being said even though she is using a CI and is relying on lipreading to understand speech. One participant stated that she had some difficulties even in quiet situations and the remaining three had some difficulties hearing speech, especially in noisy situations. Three of the subjects used a telephone every day and two used it weekly. Four of the participants used either hearing aid with a T-switch or a CI when using the phone while the fifth participant used a telephone with amplification.

\section{Results}

\subsection{British Lab-Based and Swedish Evaluations}

The evaluation results from the British lab-based tests and the Swedish tests have been combined in the following. Overall the participants found that Synface gave 
some support. In general attitudes to Synface varied with $17 \%$ finding Synface helped a lot, $65 \%$ finding Synface helped a little and $19 \%$ finding it did not help. Overall only $8 \%$ of the participants stated that they felt that Synface did not make communication clearer when using a voice telephone and only $6 \%$ stated that they felt Synface did not make communication more effective. When participants were asked before using the prototype if they felt that Synface could help them use the telephone more successfully, responses were all generally positive or neutral. This suggests that if the prototype was improved, there is the potential for Synface to assist many hard of hearing people to use a voice telephone more successfully. This suggestion is also supported by the $80 \%$ of participants who used the Synface prototype and stated that it was useful product and the $63 \%$ that stated that it was an enjoyable way to communicate. The questionnaires asked participants about problems that they encountered while using the Synface prototype. A large number noticed that Synface made errors, although there was slight variation in the number of errors noticed. The errors that were noticed were found to only upset or annoy $38 \%$ of participants but caused confusion for $54 \%$ of participants. Comments made after the UK home based evaluations suggest that with time participants may be able to learn to accommodate some of these errors. Each of the evaluations also highlighted that participants showed a preference for a more realistic face and $67 \%$ of participants stated that they felt that the movements of the face were not natural. However other qualitative comments made by participants could suggest that these responses were due to the fact that facial expressions indicating for example agreement, turntaking, mode and emotions, etc. were not available with Synface; that there is a need for greater articulation and differentiation between the visual aspect of the Synface prototype; or that as suggested in the UK evaluations, preference for a more natural or realistic face may be due to personal preference, and not relate to comprehension. When asked if they would prefer Synface to other telephone alternatives, participants indicated that they preferred Synface, and that a videophone was more popular than using a textphone.

\subsection{Comprehension Experiments}

A comprehension test was included in the Swedish evaluations. In this test comparisons were made between presenting speech as audio only, through Synface or through a talking head with hand edited recognition or as a video recording, Fig.2. The test material has been used in an earlier study 6]. Most users gained some help from Synface compared to no face. Lipreading a real face was found to be more beneficial to comprehension than Synface. This is probably due to the fact that Synface does not display facial expressions to signal agreement, turn-taking etc., which is present with a real face.

\subsection{Home-Based British Evaluations}

These evaluations have further highlighted the potential of Synface to improve communication for hard or hearing people. The results suggest that some benefit 
was gained when using Synface. After extended use participants commented that more benefits were gained once they accepted Synface was an aid to listening rather than a replacement, and with time they were able to accommodate some of the errors that were consistently made by the prototype. No learning effects were found to occur over the duration of the evaluations ( 2 weeks). This may have been due to the length of the evaluation, the measures used or that this skill requires more time to be learnt. However more research would be needed before further conclusions could be drawn.

The simplicity of the prototype allowed participants to interact with the technology with minimal distractions, the importance of which was highlighted by the increased levels of concentration that participants felt were needed using Synface compared to a standard voice telephone. A simple prototype also allowed the investigation of users attitudes towards the concept of a lip readable telephone rather than specific features of the prototype. Although participants showed a preference for a more realistic face during the lab based evaluations, this was seen to be less important after extended use. However a more realistic or natural looking face would be more enjoyable to look at.

After using Synface for two weeks, results highlight the importance of the lip movements being as accurate as possible and that animations are in sync with the audio. Comments were also made that these lip movements need to be emphasised so that it is easy to distinguish between different sounds. The switching mechanism that is used to prevent the delayed audio being played back to participants was also noticed more frequently with extended use and was still seen as not being ideal by the majority of participants.

Despite a number of participants who commented that they made more calls than they would normally whilst using Synface, they felt that once Synface was removed they would quickly slip back into their old ways. Synface did effect how participants communicated, and that with a number of improvements lipreadable devices such as Synface may be desirable and valuable pieces of technology, improving the chances for hard of hearing people to have equal access to voice communication.

\section{Conclusions and Future Work}

From the results of the evaluations it is difficult to indicate exactly who found Synface the most or least beneficial. Lipreading ability was not found to relate to those who benefited from Synface. However, the UK lab and the Swedish results appear to suggest that those participants, who are able to use a standard voice telephone successfully, gained less benefit from Synface than those who encounter greater problems. The UK evaluations also suggest that those participants who struggle to use a voice telephone still struggle when using Synface. A number of participants, including those who gained very little benefit from using Synface in each of the countries, also stated that Synface was useful with specific details such as names and numbers that they normally struggle with. 
The Synface prototype is currently undergoing usability improvements, and a pre-release version of Synface for IP-telephony will be offered to hard of hearing users in Sweden and UK during spring 2006, by newly founded company SynFace AB http://www.synface.com.

\section{Acknowledgements}

The SYNFACE project is financed by the European Union (EU) under the FP5 IST Key Action I Programme: Systems and Services for the Citizen. The project also benefits from an equipment donation within the HP VoiceWeb Initiative.

In addition to the authors the following people have been active in the SYNFACE project: B. Granström, K-E Spens at KTH, A. Faulkner, C. Siciliano, G. Williams at UCL, UK, J. Kardach, E. Landström, J-I Lindström at Acapela Sweden AB, Sweden, M. Sheard at RNID, UK, and B. Elsendoorn, E. Rikken, N. van Son, A vWijk at Viataal, the Netherlands.

\section{References}

1. Lönnborg, T.: Mobil videotelefoni som kommunikationshjälpmedel för individer med hörselnedsättning. Master's thesis, Linköpings universitet (2005)

2. Beskow, J., Karlsson, I., Kewley, J., Salvi, G.: SYNFACE - a talking head telephone for the hearing-impaired. In Miesenberger, K., Klaus, J., Zagler, W., Burger, D., eds.: Computers Helping People with Special Needs, Springer (2004) 1178-1186

3. Salvi, G.: Truncation error and dynamics in very low latency phonetic recognition. In: ISCA workshop on Non-linear Speech Processing. (2003)

4. Beskow, J.: Animation of talking agents. In: Proc. of AVSP'97, Rhodes (1997)

5. Beskow, J.: Trainable articulatory control models for visual speech synthesis. Journal of Speech Technology 7(4) (2004) 335-349

6. Siciliano, C., Williams, G., Beskow, J., Faulkner, A.: Evaluation of a multilingual synthetic talking face as a communication aid for the hearing impaired. In: Proc. of ICPhS. (2003) 131-134 